\title{
Tratamiento del hipoparatiroidismo con hormona paratiroidea
}

\section{Treatment of hypoparathyroidism with parathyroid hormone}

\author{
Esteban Cardona ${ }^{1}$, Carlos Alfonso Builes-Barrera², Alejandro Román-González ${ }^{2}$
}

${ }^{1}$ Estudiante de Medicina, Universidad de Antioquia, Medellin, Colombia.

${ }^{2}$ Profesor, Departamento de Medicina Interna, Universidad de Antioquia. Internista Endocrinólogo, Hospital Universitario San Vicente Fundación, Medellín, Colombia.

Correspondencia: Dr. Alejandro Román-González

Email: alejoroman@gmail.com

Fecha de recepción: 18/06/2018

Fecha de aceptación: 17/07/2018

\section{Resumen}

El hipoparatiroidismo es un trastorno del metabolismo del calcio infrecuente. La principal etiología es la posquirúrgica en el $75 \%$ de los casos. El hipoparatiroidismo crónico tiene una prevalencia estimada de 20-40 por cada 100.000 habitantes. Las manifestaciones clínicas varían desde cuadros asintomáticos hasta neuromusculares, neurológicas, cognitivas, cardiacas y disminución en la calidad de vida. El tratamiento estándar es la suplementación de calcio y calcitriol. Es frecuente la refractariedad a este enfoque terapéutico y se asocia a una alta incidencia de efectos adversos por las altas dosis de suplementos requeridas. Hasta hace poco era la única deficiencia endocrina no tratada con terapia de sustitución hormonal. Recientemente, la FDA ha aprobado el uso de la PTH recombinante (1-84) para el tratamiento del hipoparatiroidismo refractario. Hay dos análogos de PTH disponibles (PTH 1-84 y PTH 1-34). Ambas moléculas se han asociado a una disminución en los requerimientos de suplementación con calcio y calcitriol, manteniendo concentraciones estables de calcio sérico y fosfato, mejor calidad de vida y un metabolismo óseo más fisiológico con una baja incidencia de efectos adversos. Actualmente, la terapia de sustitución hormonal con PTH no hace parte del tratamiento de rutina del hipoparatiroidismo y tiene unas indicaciones estrictas.

Palabras claves: Hipoparatiroidismo, hormona paratiroidea, PTH (1-34), PTH (1-84), teriparatida

\section{Abstract}

Hypoparathyroidism is an uncommon disorder of calcium metabolism. The main etiology is post-surgical in $75 \%$ of cases. Chronic hypoparathyroidism has an estimated prevalence of 2040 per 100,000 inhabitants. Clinical manifestations vary from asymptomatic to neuromuscular, neurological, cognitive, cardiac and decrease in quality of life. The standard treatment is calcium and calcitriol supplementation. Refractoriness to this therapeutic approach is frequent and is associated with a high incidence of adverse effects due to the high doses of supplements required. Until recently it was the only endocrine deficiency not treated with hormone replacement therapy. Recently the FDA has approved the use of recombinant PTH (1-84) for the treatment of refractory hypoparathyroidism. There are two PTH analogues available (PTH 1-84 and PTH 1-34). Both molecules have been associated with a decrease in the requirements of calcium and calcitriol supplementation, maintaining stable concentrations of serum calcium and phosphate, better quality of life and a more physiological bone metabolism with a low incidence of adverse effects. Currently hormone replacement therapy with PTH is not part of the routine treatment of hypoparathyroidism and has strict indications.

Key words: Hypoparathyroidism, parathyroid hormone, PTH (1-34), PTH (1-84), teriparatide.

\section{Introducción}

El hipoparatiroidismo es un trastorno del metabolismo del calcio con una baja prevalencia en la población general ${ }^{(1)}$. Genera alteraciones en el metabolismo óseo, en variables bioquímicas como el calcio y el fosfato y un impacto en la calidad de vida de las personas ${ }^{(2)}$. El tratamiento estándar con suplementación de calcio y calcitriol se asocia con una alta incidencia de efectos adversos debido a las altas dosis requeridas ${ }^{(3)}$. Es frecuente la refractariedad a estas medidas terapéuticas ${ }^{(4,5)}$. Hasta hace poco era la única deficiencia endocrina sin tratamiento de sustitución hormonal. Recientemente, la FDA aprobó la terapia de remplazo con PTH recombinante (1-84) para el tratamiento del hipoparatiroidismo refractario ${ }^{(6)}$. Esta revi- 
sión pretende evaluar la evidencia en relación con la eficacia, seguridad e indicaciones de análogos de PTH en el manejo del hipoparatiroidismo.

\section{Generalidades del hipoparatiroidismo}

El hipoparatiroidismo es un trastorno endocrino del metabolismo de calcio poco frecuente, caracterizado por concentraciones inapropiadamente bajas de hormona paratiroidea (PTH), hipocalcemia, hiperfosfatemia, alteraciones del metabolismo óseo y disminución en la calidad de vida ${ }^{(7,8)}$. Estas alteraciones son las responsables de las manifestaciones clínicas asociadas a la enfermedad.

El hipoparatiroidismo adquirido es el más común. En el $75 \%$ de los casos es secundario a intervenciones quirúrgicas como consecuencia de la resección de la glándula tiroides o lesión secundaria a una intervención cervical ${ }^{(9,10)}$. La incidencia del hipoparatiroidismo posquirúrgico está ligada a la extensión de la intervención y a la experiencia del cirujano ${ }^{(11)}$.

La prevalencia estimada en EE.UU de hipoparatiroidismo crónico es de 37 por cada 100.000 habitantes (59.000$115.000)$ y se clasifica como una enfermedad huérfana ${ }^{(12)}$. Veintinueve de cada 100.000 habitantes son de etiología posquirúrgica. En Dinamarca se reporta una prevalencia estimada de hipoparatiroidismo crónico de aproximadamente 24 por cada 100.000 habitantes y de 22 de cada 100.000 habitantes de etiología posquirúrgica. La incidencia estimada es de 0,8 por cada 100.000 habitantes año ${ }^{(13,14)}$. Un estudio realizado en Escocia reporta una prevalencia estimada de hipoparatiroidismo crónico de 40 por cada 100.000 habitantes, con una tasa de 23 y 17 por cada 100.000 habitantes para etiología posquirúrgica y no quirúrgica, respectivamente ${ }^{(15)}$.

Las manifestaciones clínicas se deben principalmente a la hipocalcemia e hiperfosfatemia crónica; varía desde cuadros asintomáticos hasta manifestaciones neuromusculares leves, disfunción neurológica, cognitiva, muscular, cardiaca y calcificaciones ectópicas de tejidos blandos ${ }^{(8,16,17)}$. Hay manifestaciones esqueléticas caracterizadas por alteraciones en la densidad mineral ósea (DMO), disminución en la porosidad, bajo recambio óseo y un riesgo potencial de fractura no bien determinado y disminución en la calidad de vida(18-20).

El tratamiento estándar es la suplementación de calcio y análogos de vitamina $\mathrm{D}$ activa. Los objetivos del tratamiento son la disminución y prevención de signos y síntomas de hipocalcemia, mantenimiento el calcio en el rango inferior normal, el producto calcio/fósforo menor de $55 \mathrm{mg}^{2} / \mathrm{dl}^{2}$, evitar la hipercalciuria, la hipercalcemia y las calcificaciones en tejidos blandos ${ }^{(7,9)}$.

Frecuentemente, la enfermedad es refractaria al tratamiento o son necesarias dosis altas para lograr los objetivos; esto se asocia a una alta incidencia de efectos adversos graves como nefrocalcinosis, nefrolitiasis y calcificación de tejidos blandos. Este enfoque terapéutico está limitado por el control moderado del trastorno endocrino y las potenciales consecuencias de las altas dosis de suplementación ${ }^{(4,7)}$.

Hasta hace poco fue la única deficiencia endocrina no tratada con terapia de sustitución hormonal. Sin embargo, desde 1929, el doctor Fuller Albright había usado extractos de paratiroides en un paciente con hipoparatiroidismo idiopático ${ }^{(21)}$. Debido a que la terapia convencional a menudo no es efectiva y a la alta incidencia de efectos adversos, el uso de la PTH constituye una alternativa atractiva como un tratamiento más fisiológico de esta deficiencia endocrina. En el 2015, la FDA aprobó el uso de la PTH recombinante (1-84) para el tratamiento del hipoparatiroidismo refractario. Actualmente, la PTH no hace parte del tratamiento de rutina para el hipoparatiroidismo y sus indicaciones son estrictas ${ }^{(9)}$.

\section{Hormona paratiroidea}

Existen dos moléculas disponibles como análogos de la PTH, la PTH 1-34 (Teriparatida) y la PTH 1-84 (Natpara). La PTH 1-84 tiene una vida media más larga que permite un intervalo de dosificación más amplio. La concentración plasmática presenta una curva bifásica, con niveles máximos a los 15 minutos y a las 2 horas posteriores a la administración subcutánea, en contraste con una concentración pico monofásico con la administración intravenosa. El calcio sérico no se correlaciona con las concentraciones pico de PTH, una exposición constante a dosis bajas puede ser más eficaz en el mantenimiento de la homeostasis de calcio que una dosis alta con menor frecuencia de administración. Los usos de dosis bajas con mayores frecuencias reducen la dosis total necesaria de $\mathrm{PTH}^{(5)}$. La concentración de PTH es indetectable 12 a 24 horas posteriores a la dosificación. El efecto en los niveles plasmáticos de calcio, fosfato y calcitriol $(1,25(\mathrm{OH}) 2 \mathrm{D})$ dura 24 horas, por lo cual la dosificación diaria es preferible. La PTH actúa rápidamente en el túbulo renal, reduciendo el fosfato sérico y más tardíamente en el sistema óseo y la 1 alfa hidroxilasa renal, aumentando el calcio sérico. Se deben medir niveles plasmáticos de calcio aproximadamente 7 horas después de la administración, para ajustar la dosis y evitar la hipercalcemia ${ }^{(5,22)}$. La PTH 1-34 tiene una vida media corta, el intervalo de dosificación debe ser más corto o con sistemas de bomba de infusión ${ }^{(23)}$.

\section{Efecto de PTH en variables bioquímicas}

La administración de PTH permite la reducción de los suplementos de calcio y análogos de vitamina $\mathrm{D}$ activa, manteniendo concentraciones séricas de calcio en el rango inferior de la normalidad. En la tabla 1 se presenta un resumen de los estudios más importantes de PTH en el manejo del hipoparatiroidismo.

En el estudio REPLACE, la administración de hormona paratiroidea recombinante humana (PTH 1-84) permitió la reducción de más del $50 \%$ en la suplementación de calcio y 
Tabla 1. Ensayos clínicos de PTH en el tratamiento del hipoparatiroidismo

\begin{tabular}{|c|c|c|c|c|c|c|c|c|}
\hline Autor- año & PTH & Muestra & Seguimiento & $\begin{array}{l}\text { Reducción } \\
\text { calcio oral }\end{array}$ & $\begin{array}{l}\text { Reducción } \\
\text { calcitriol }\end{array}$ & $\begin{array}{l}\text { Eliminación } \\
\text { calcitriol }\end{array}$ & $\begin{array}{c}\text { Densidad mineral } \\
\text { ósea }\end{array}$ & Otros resultados \\
\hline $\begin{array}{l}\text { Sikjaer T, et al } \\
2011(40)^{*}\end{array}$ & $1-84$ & 62 & 24 semanas & $47 \%(f)$ & $50 \%$ & $22 \%(c)$ & $\begin{array}{c}C L+12,2 \% \\
\text { CT }-2,3 \%\end{array}$ & $\begin{array}{l}\text { Sin beneficio en } \\
\text { calidad de vida y } \\
\text { función muscular }\end{array}$ \\
\hline $\begin{array}{l}\text { Clarke BL, et al } \\
2017(26)\end{array}$ & $1-84$ & $\begin{array}{c}\text { PTH (80) } \\
\text { Placebo (44) }\end{array}$ & 24 semanas & $52 \%$ & $\begin{array}{l}77 \pm 4 \% \\
87 \%(b)\end{array}$ & $62 \%$ & $\mathrm{~N} / \mathrm{A}$ & N/A \\
\hline $\begin{array}{l}\text { Clarke BL, et al } \\
2014(5)\end{array}$ & $1-84$ & 8 & N/A\$ & $\mathrm{N} / \mathrm{A}$ & $\mathrm{N} / \mathrm{A}$ & $\mathrm{N} / \mathrm{A}$ & $\mathrm{N} / \mathrm{A}$ & $\begin{array}{l}\text { Metabolismo de } \\
\text { calcio, magnesio, } \\
\text { fosfato y vitamina D }\end{array}$ \\
\hline $\begin{array}{l}\text { Rubin MR, et al } \\
2016(25)\end{array}$ & $1-84$ & 33 & 6 años & $53 \%$ & $67 \%$ & $48 \%$ & $\begin{array}{l}\mathrm{CL}+3,8 \pm 1 \% \\
\mathrm{CT}+2,4 \pm 1 \% \\
\mathrm{CF}+2,6 \pm 1 \% \\
\mathrm{RD}-4.4 \pm 1 \% .\end{array}$ & N/A \\
\hline $\begin{array}{l}\text { Cusano NE, et al } \\
2013(27)\end{array}$ & $1-84$ & 27 & 4 años & $37 \%$ & $45 \%$ & $26 \%$ & $\begin{array}{c}C L+5,5 \pm-9 \% \\
C T+1,1 \pm 9 \% \\
C F+0,6 \pm 12 \% \\
\text { RD sin cambios }\end{array}$ & N/A \\
\hline $\begin{array}{l}\text { Cusano Ne, et al } \\
\text { 2014(32) }\end{array}$ & $1-84$ & 69 & 5 años & $\mathrm{N} / \mathrm{A}$ & $\mathrm{N} / \mathrm{A}$ & $\mathrm{N} / \mathrm{A}$ & $\mathrm{N} / \mathrm{A}$ & $\begin{array}{l}\text { Mejoría salud mental y } \\
\text { física }(d)\end{array}$ \\
\hline $\begin{array}{l}\text { Lakatos P, et al } \\
2016(28)\end{array}$ & $1-84$ & 24 & 24 semanas & $\begin{array}{c}76 \% \\
71 \%(a)\end{array}$ & $97 \%$ & $58 \%$ (c) & $\mathrm{N} / \mathrm{A}$ & N/A \\
\hline $\begin{array}{l}\text { Mannstadt M, et } \\
\text { al 2013(24) }\end{array}$ & $1-84$ & $\begin{array}{c}\text { PTH (90) } \\
\text { Placebo (44) }\end{array}$ & 24 semanas & $\begin{array}{c}52 \% \\
53 \%(a)\end{array}$ & $78 \%$ & $\mathrm{~N} / \mathrm{A}$ & $\mathrm{N} / \mathrm{A}$ & N/A \\
\hline $\begin{array}{l}\text { Rubin MR, et al } \\
\text { 2010(41) }\end{array}$ & $1-84$ & 30 & 24 meses & $\begin{array}{c}\text { Disminución } \\
\text { de } 3.030 \pm \\
2.325 \text { a } 1.661 \pm \\
1.267 \mathrm{mg} / \text { día }\end{array}$ & $41 \%$ & $27 \%$ & $\begin{array}{c}\mathrm{CL}+2,9 \pm 4 \% \\
\mathrm{CF} \text { sin cambios } \\
\mathrm{RD}-2,4 \pm 4 \%\end{array}$ & N/A \\
\hline $\begin{array}{l}\text { Rubin MR, et al } \\
2011(42)\end{array}$ & $1-84$ & 64 & 24 meses & $\mathrm{N} / \mathrm{A}$ & N/A & N/A & $\mathrm{N} / \mathrm{A}$ & $\begin{array}{l}\text { Aumento índice } \\
\text { histomorfométricos } \\
\text { y bioquímicos del } \\
\text { metabolismo óseo }\end{array}$ \\
\hline $\begin{array}{l}\text { Bilezikian JP, et } \\
\text { al 2017(43) }\end{array}$ & $1-84$ & $\begin{array}{l}25 \mu \mathrm{g} / \mathrm{d}(19) \\
50 \mu \mathrm{g} / \mathrm{d}(23)\end{array}$ & 8 semanas & $\begin{array}{c}7 \%(25 \mu \mathrm{g} / \mathrm{d}) \\
21 \%(\mathrm{e}) \\
10 \%(50 \mu \mathrm{g} / \mathrm{d}) \\
26 \%(\mathrm{e})\end{array}$ & $\begin{array}{c}3 \%(25 \\
\mu g / d), 11 \% \\
(\text { a) } 39 \%(50 \\
\mu g / d), 26 \% \\
\text { (a) }\end{array}$ & N/A & $\mathrm{N} / \mathrm{A}$ & N/A \\
\hline $\begin{array}{l}\text { Vokes et al } \\
2018(44)\end{array}$ & $1-84$ & $\begin{array}{c}\text { PTH (83) } \\
\text { Placebo (39) }\end{array}$ & 24 semanas & N/A & $\mathrm{N} / \mathrm{A}$ & N/A & $\mathrm{N} / \mathrm{A}$ & $\begin{array}{l}\text { Mejoría componente } \\
\text { físico, dolor corporal y } \\
\text { vitalidad (d) }\end{array}$ \\
\hline $\begin{array}{l}\text { Palermo A, et al } \\
2018(29)^{* *}\end{array}$ & $1-34$ & 42 & 24 meses & $72 \%$ & $\begin{array}{c}64 \% \\
(6 \text { meses) } \\
49 \% \\
(2 \text { años) }\end{array}$ & $\mathrm{N} / \mathrm{A}$ & $\mathrm{N} / \mathrm{A}$ & $\begin{array}{l}\text { Mejora de los } 8 \\
\text { dominios (d) }\end{array}$ \\
\hline $\begin{array}{l}\text { Gutiérrez LE, et } \\
\text { al } 2016\end{array}$ & $1-34$ & 16 & 3 meses & $\begin{array}{c}78 \% \\
100 \%(b)\end{array}$ & $\begin{array}{c}44,7 \% \\
68 \%(b)\end{array}$ & $\mathrm{N} / \mathrm{A}$ & $\mathrm{N} / \mathrm{A}$ & N/A \\
\hline $\begin{array}{l}\text { Winer KK, et al } \\
\text { 2012(23) }\end{array}$ & $1-34$ & 8 & 6 meses & $\mathrm{N} / \mathrm{A}$ & $\mathrm{N} / \mathrm{A}$ & $\mathrm{N} / \mathrm{A}$ & $\mathrm{N} / \mathrm{A}$ & $\begin{array}{l}\text { Reducción } 65 \% \text { dosis } \\
\text { PTH (administración } \\
\text { bomba de insulina) }\end{array}$ \\
\hline
\end{tabular}




\begin{tabular}{l|c|c|c|c|c|c|c|c}
\hline $\begin{array}{l}\text { Winer KK, et al } \\
2010(45)\end{array}$ & $1-34$ & 12 & 3 años & N/A & N/A & N/A & En rango normal & $\begin{array}{l}\text { Niveles de calcio sérico } \\
\text { en rango normal sin } \\
\text { hipercalciuria }\end{array}$ \\
\hline $\begin{array}{l}\text { Winer KK, et al } \\
2008(46)\end{array}$ & $1-34$ & 14 & 28 semanas & N/A & N/A & N/A & $\begin{array}{l}\text { N/A } \\
\text { menor variación sérica } \\
\text { de calcio (2 dosis/ } \\
\text { día) }\end{array}$ \\
\hline $\begin{array}{l}\text { Winer KK, et al } \\
2003(47)\end{array}$ & $1-34$ & 27 & 3 años & N/A & N/A & N/A & N/A & $\begin{array}{l}\text { Niveles normales } \\
\text { de calcio sérico sin } \\
\text { hipercalciuria }\end{array}$ \\
\hline $\begin{array}{l}\text { Winer KK, et al } \\
1998(48)\end{array}$ & $1-34$ & 17 & 28 semanas & N/A & N/A & N/A & N/A & $\begin{array}{l}\text { Reducción dosis PTH y } \\
\text { menor variación sérica } \\
\text { de calcio (2 dosis/ } \\
\text { día)*** }\end{array}$ \\
\hline $\begin{array}{l}\text { Winer KK, et al } \\
1996(49)\end{array}$ & $1-34$ & 10 & 20 semanas & N/A & N/A & N/A & N/A & $\begin{array}{l}\text { Calcio sérico en rango } \\
\text { normal, disminución } \\
\text { de calciuria. Aumento } \\
\text { de marcadores de } \\
\text { recambio óseo }\end{array}$ \\
\hline
\end{tabular}

(*) Incluye publicaciones de Sikjaer $\mathrm{T}$, et al. en $2011^{(40)}, 2012^{(50)}, 2013^{(22)}$ y $2014^{(51)}$ que analizaron diferentes resultados a partir del mismo ensayo clínico. ${ }^{* *}$ ) Incluye resultados de Santonati A, 2015 $5^{(52)}$.

$\left.{ }^{* * *}\right)$ Comparación régimen de tratamiento 2 dosis/día con 1 dosis/día.

(\$) Estudio de propiedades farmacológicas de PTH, dosis única de tratamiento

a. Pacientes con reducción $\geq 50 \%$ en calcio oral y calcitriol.

b. Pacientes con reducción $\geq 50 \%$ en la dosis.

c. Pacientes que eliminaron suplementación de calcitriol y calcio.

d. Calidad de vida (cuestionario SF-36)

e. Reducciones suplementación de calcio $\leq 500 \mathrm{mg} /$ día y calcitriol $\leq 0,25 \mu \mathrm{g} / \mathrm{d}$

f. Pacientes que eliminaron suplementación con calcio.

Abreviaturas:

CL: columna lumbar

CT: cadera total

CF: cuello femoral

RD: radio $1 / 3$ distal

análogos de vitamina D activa en 53\%, manteniendo concentraciones plasmáticas estables de calcio durante 24 semanas de tratamiento ${ }^{(24)}$.

Un estudio de 6 años de duración de seguridad y eficacia de la PTH 1-84 reportó una reducción de la suplementación de calcio en $57 \%$, vitamina D activa en $67 \%$ y la eliminación de suplementos de vitamina D activa en $48 \%$, manteniendo concentración estable de calcio sérico y disminución en la calciuria. La disminución de los requerimientos fue progresiva, este hallazgo puede deberse al cambio de dosificación de día alterno a diaria durante el estudio, sugiriendo una mayor capacidad de respuesta con este régimen de tratamiento ${ }^{(25)}$.

El tratamiento con PTH 1-84 se asocia con los efectos fisiológicos de la PTH endógena y la normalización de las variables bioquímicas, permitiendo un control sérico y urinario del calcio, disminución de fosfato sérico y del producto calcio-fósforo e incremento del recambio óseo ${ }^{(25-28)}$.

A pesar de las reducciones o eliminación en la suplementación de vitamina D activa, los pacientes tratados con PTH 1-84 mantienen niveles séricos del metabolito activo de vitamina D en rango normal, reflejando la conversión de $25(\mathrm{OH})$ a 1,25 (OH) por la PTH. En consecuencia, la administración de PTH permite la reducción de la suplementación con calcitriol, pero debe continuar la suplementación con vitamina D. En el estudio más grande de remplazo con PTH, el REPLACE, se corregía la deficiencia de vitamina $\mathrm{D}$ (menor de $30 \mathrm{ng} / \mathrm{ml}$ ) y de magnesio ( $<1,3 \mathrm{mEq} / \mathrm{L})$ antes del inicio de la PTH 1-84 ${ }^{(24,26)}$. La mediana de $25(\mathrm{OH})$ vitamina $\mathrm{D}$ al inicio del estudio fue mayor de $43 \mathrm{ng} / \mathrm{ml}$. Todos los pacientes durante el estudio se suplementaron con 400 UI de vitamina D y la dosis se aumentaba según criterio del investigador para mantener una concentración $>30 \mathrm{ng} / \mathrm{ml}^{(24)}$.

También se ha demostrado la eficacia de la PTH 1-34 en la mejora de estos parámetros, aumentando concentraciones plasmáticas de calcio y reduciendo las fluctuaciones en sus valores. Un estudio prospectivo a dos años reporta un aumento en la excreción urinaria de calcio, hallazgo que contrasta con los resultados obtenidos en los estudios de PTH 1-84; en consecuencia, se debe considerar un posible riesgo de nefrolitiasis con el uso de esta molécula ${ }^{(29)}$.

Algunas comorbilidades en el hipoparatiroidismo se deben a los altos requerimientos de suplementos con el consiguiente riesgo de calcificación ectópica; la PTH proporciona un tratamiento más fisiológico que permite una mayor seguridad que el tratamiento estándar. Ambas moléculas demuestran superioridad en el mantenimiento del calcio sérico y excreción del calcio urinario frente a la terapia convencional, con disminución de las dosis de calcio oral y vitamina $\mathrm{D}^{(25-29)}$.

\section{Dosificación}

El régimen de dosificación diaria de PTH 1-84 es más eficaz en el control del calcio, fósforo y vitamina $\mathrm{D}$, debido a que las variables bioquímicas vuelven a niveles basales 24 horas 
después de la dosificación ${ }^{(11)}$. La PTH 1-84 está disponible en rangos de dosis de 25-50-75 y $100 \mathrm{mcg} /$ día.

Se ha demostrado la eficacia con dosis de 50-75-100 mcg/ día. Una titulación con una dosis inicial de $50 \mathrm{mcg} /$ día, con incrementos de 25 mcg según la tolerancia y respuesta hasta alcanzar una dosis máxima de $100 \mathrm{mcg} /$ día reduce la incidencia de eventos hipercalcémicos ${ }^{(24,28,30)}$.

La eficacia de la PTH 1-34 ha sido reportada con dosis entre 37 y $46 \mathrm{mcg} /$ día $^{(29)}$. Debido a su corta vida media, la administración dos veces al día mantiene la eucalcemia a una dosis diaria significativamente menor que la administración una vez al día. Sin embargo, el uso en una bomba de infusión ha demostrado una reducción adicional de la dosis y una menor fluctuación en el calcio sérico, proporcionando una terapia de remplazo más fisiológica ${ }^{(23)}$.

\section{Calidad de vida}

El hipoparatiroidismo se asocia a una disminución en la calidad de vida, independiente de la etiología, duración y control bioquímico del trastorno endocrino. Se ha asociado la hipocalcemia crónica con alteraciones cognitivas, depresión, ansiedad y trastornos de personalidad que influyen en el deterioro de la calidad de vida ${ }^{(2,3,8,16,18,31)}$.

El uso de PTH 1-84 ha demostrado una mejor percepción de la salud mental y física, variables evaluadas en las escalas de calidad de vida. Estos efectos se observan a corto y largo plazo $^{(32)}$. La concentración de calcio sérico en rangos ideales, la disminución en la fluctuación de sus valores y la reducción en las dosis de suplementación logrados con la terapia de remplazo hormonal parecen explicar el aumento del bienestar físico y mental. Estos hallazgos podrían ser explicados por un mecanismo directo de la PTH o indirectamente por un mayor control clínico y una disminución en la suplementación que es incómodo para los pacientes. El reporte de una menor puntuación en la calidad de vida en el tratamiento con PTH 1-34 que exige una mayor frecuencia de dosificación está a favor de un mecanismo indirecto ${ }^{(29)}$.

\section{Seguridad}

La PTH para el tratamiento de la osteoporosis fue aprobada con una advertencia de riesgo potencial de osteosarcoma, evidencia que se encontró en ratones. Esto limita el tratamiento con PTH en osteoporosis a una duración máxima de dos años. Este hallazgo fue dependiente de la dosis y la duración del tratamiento. Adicionalmente, existen diferencias marcadas en la fisiología ósea entre ratones y humanos. En diferentes estudios no se ha detectado asociación entre el tratamiento con teriparatida y osteosarcoma en humanos ${ }^{(33,34)}$.

El uso de la PTH 1-84 se asocia con una menor incidencia de signos y síntomas de hipocalcemia ${ }^{(35)}$. Sin embargo, el riesgo de desarrollarla es elevado al suspender la terapia con
PTH y son necesarias dosis mayores de suplementos de calcio y análogos de vitamina $\mathrm{D}$ activa que las previas al tratamiento con PTH 1-84(24).

La hipercalcemia es el evento adverso grave más común, aunque es poco frecuente. Se ha reportado en $2,5 \%$ de las mediciones de calcio sérico en un seguimiento a seis años ${ }^{(25)}$. Una individualización en el régimen de dosificación reduce la incidencia de hipercalcemia ${ }^{(22)}$. En un estudio de eficacia y seguridad de seis años de duración, los efectos adversos más comunes fueron dolores musculoesqueléticos ${ }^{(25)}$. Los episodios de hipercalcemia reportados no requirieron hospitalización ${ }^{(24)}$.

Se han reportado hallazgos similares con PTH 1-34. El efecto adverso más común es la hipercalcemia asintomática (eventos en $7 \%$ de los pacientes) asociada a una lenta reducción de la suplementación de calcio y análogos de vitamina $\mathrm{D}$ activa. Ningún evento de hipercalcemia requirió hospitalización. Los efectos adversos leves como mialgia y síntomas gastrointestinales se resolvieron con la interrupción del tratamiento. En contraste, con el uso de la PTH 1-84, se reportaron episodios asintomáticos de hipercalciuria ${ }^{(29)}$.

No se han informado cambios significativos en variables cardiovasculares como presión arterial, frecuencia cardiaca o intervalo QTc o en variables renales con la terapia de sustitución de $\mathrm{PTH}^{(24)}$.

Cusano y colaboradores reportaron la recuperación de la función paratiroidea en cuatro pacientes con un diagnóstico previo de hipoparatiroidismo de 8 a 16 años $^{(36)}$. La recuperación se asoció con la administración de PTH 1-84 durante 3 a 5 años. Se plantea un mecanismo mediado por la alteración en los niveles del factor de crecimiento endotelial vascular ${ }^{(37)}$. Sin embargo, se desconocen los mecanismos por los cuales los individuos recuperaron la función de las glándulas paratiroides.

\section{Densidad mineral ósea y recambio óseo}

En la experiencia clínica de tratamiento con PTH 1-84 más larga hasta el momento, hubo un aumento en la DMO en la columna lumbar y cadera total. El aumento reportado fue progresivo con una ganancia promedio de $3,8 \pm 1 \%$ para columna lumbar y $2,4 \pm 1 \%$ para cadera total a los seis años. La DMO disminuyó en el tercio del radio distal con una pérdida media de 4,4 $\pm 1 \%$. La DMO del cuello femoral aumentó al año 3 , pero fue igual a la línea de base al final del estudio ${ }^{(25)}$. Estos resultados reflejan el efecto diferencial de la PTH en los sitios que son predominantemente hueso cortical o trabecular. Los marcadores de recambio y formación ósea aumentaron, con valores más elevados durante el primer año de tratamiento seguido de un descenso a niveles que permanecieron por encima del rango de referencia. No está claro si los cambios en la DMO están asociados con un mayor riesgo de fractura en hueso cortical o proporcionan ventajas biomecánicas a pesar de la disminución en la DMO. En contraste con la osteoporosis, algunos 
autores han propuesto que una disminución en la DMO en el hipoparatiroidismo puede ser saludable ${ }^{(7)}$. Sin embargo, esto es controversial y aún faltan estudios a largo plazo que nos aclaren este aspecto. Por ende, las implicaciones de estos cambios en la DMO siguen sin determinarse ${ }^{(38)}$.

\section{Indicaciones}

Las indicaciones de restitución de PTH 1-84 son: el control variable e inconstante del calcio sérico, nefrolitiasis, nefrocalcinosis o depuración de creatinina disminuida o tasa de filtración glomerular estimada (eGFR) a $<60 \mathrm{ml} / \mathrm{min}$; hipercalciuria y/u otros índices bioquímicos de riesgo de cálculos renales; persistencia elevada de fosfato sérico y/o producto fósforo/calcio (> $55 \mathrm{mg}^{2} / \mathrm{dl}^{2}$ ); cantidades excesivas de medicamentos orales requeridos para controlar síntomas tales como $>2,5 \mathrm{~g}$ de calcio elemental $\mathrm{o}>1,5 \mu \mathrm{g}$ de vitamina $\mathrm{D}$ activa, o ambos; $\mathrm{y}$ un trastorno del tracto gastrointestinal que podría conducir a una absorción variable de calcio y vitamina $\mathrm{D}^{(7,9)}$. Se recomienda iniciar con dosis de $50 \mathrm{mcg} /$ día vía subcutánea $^{(24)}$. Simultáneamente, reducir la dosis de vitamina D activa en un $50 \%$. La concentración sérica de calcio se controla dentro de la primera semana de inicio y cada vez que se cambia la dosis de PTH 1-84. Los objetivos son minimizar o eliminar el uso de vitamina D activa, reducir el suplemento de calcio elemental a $500 \mathrm{mg}$ diarios y mantener el calcio sérico en el rango inferior de lo normal. Actualmente, PTH 1-34 no está aprobada para el tratamiento del hipoparatiroidismo. En un estudio preliminar en Colombia de pacientes con hipoparatiroidismo se encontró que 4,1\% de los pacientes estaban siendo tratados con $\mathrm{PTH}^{(39)}$, lo que refleja que es una terapia para pacientes muy seleccionados.

\section{Financiación}

Trabajo financiado en parte por la Asociación Colombiana de Endocrinología, Diabetes y Metabolismo, Hospital Universitario San Vicente Fundación y Universidad de Antioquia.

\section{Posibles conflictos de interés:}

Esteban Cardona: Ninguno

Alejandro Román-González: He participado en cenas, congresos, advisory boards o conferencias patrocinadas y/o he recibido patrocinio económico de las siguientes compañías: Sanofi, Amgen, Novartis, Shire, Colciencias, Valentech, Laboratorio Investigación Hormonal, Novo Nordisk, Merck, Hospital Universitario San Vicente Fundación, Universidad de Antioquia y Asociación Colombiana de Endocrinología, Diabetes y Metabolismo, Universidad del Bosque.

Carlos Alfonso Builes-Barrera: He participado en cenas, congresos, advisory boards o conferencias patrocinadas y/o he recibido patrocinio económico de las siguientes compañías: Sanofi, Amgen, Lilly, Novartis, Laboratorio Investigación Hormonal, Merck, Abbott, Dinámica IPS, Hospital Universitario San Vicente Fundación, Universidad de Antioquia y Asociación Colombiana de Endocrinología, Diabetes y Metabolismo.

\section{Referencias}

1. Zea Lopera J, Londoño Tabares S, Builes-Barrera C, Sanabria A, RománGonzález, A. Pilares para el enfoque y tratamiento adecuado del paciente con hipoparatiroidismo. Iatreia. 2018;31(2):155-65.

2. Büttner M, Musholt TJ, Singer S. Quality of life in patients with hypoparathyroidism receiving standard treatment: a systematic review. Endocrine. 2017;58(1):14-20.

3. Arlt W, Fremerey C, Callies F, Reincke M, Schneider P, Timmermann W, et al. Well-being, mood and calcium homeostasis in patients with hypoparathyroidism receiving standard treatment with calcium and vitamin D. Eur J Endocrinol. 2002;146(2):215-22.

4. Stack BC, Bimston DN, Bodenner DL, Brett EM, Dralle H, Orloff LA, et al. American Association Of Clinical Endocrinologists And American College Of Endocrinology Disease State Clinical Review: Postoperative Hypoparathyroidism--Definitions And Management. Endocr Pract. 2015;21(6):674-85.
5. Clarke BL, Kay Berg J, Fox J, Cyran JA, Lagast H. Pharmacokinetics and pharmacodynamics of subcutaneous recombinant parathyroid hormone (1-84) in patients with hypoparathyroidism: an open-label, single-dose, phase I study. Clin Ther. 2014;36(5):722-36.

6. Rejnmark L, Underbjerg L, Sikjaer T. Hypoparathyroidism: Replacement Therapy with Parathyroid Hormone. Endocrinol Metab (Seoul). 2015;30(4):436-42.

7. Bilezikian JP, Brandi ML, Cusano NE, Mannstadt M, Rejnmark L, Rizzoli R, et al. Management of Hypoparathyroidism: Present and Future. J Clin Endocrinol Metab. 2016;101(6):2313-24.

8. Shoback DM, Bilezikian JP, Costa AG, Dempster D, Dralle H, Khan AA, et al Presentation of Hypoparathyroidism: Etiologies and Clinical Features. J Clin Endocrinol Metab. 2016;101(6):2300-12.

9. Brandi ML, Bilezikian JP, Shoback D, Bouillon R, Clarke BL, Thakker RV, et al. 
Management of Hypoparathyroidism: Summary Statement and Guidelines. J Clin Endocrinol Metab. 2016;101(6):2273-83.

10. Bilezikian JP, Khan A, Potts JT, Brandi ML, Clarke BL, Shoback D, et al. Hypoparathyroidism in the adult: epidemiology, diagnosis, pathophysiology, target-organ involvement, treatment, and challenges for future research. ] Bone Miner Res. 2011;26(10):2317-37.

11. Cocchiara G, Cajozzo M, Fazzotta S, Palumbo VD, Geraci G, Maione C, et al. [Risk factors' analysis of transient and permanent hypoparathyroidism after thyroidectomy]. Clin Ter. 2017;168(4):e271-e7.

12. Powers J, Joy K, Ruscio A, Lagast H. Prevalence and incidence of hypoparathyroidism in the United States using a large claims database. J Bone Miner Res. 2013;28(12):2570-6.

13. Clarke BL, Brown EM, Collins MT, Jüppner H, Lakatos P, Levine MA, et al. Epidemiology and Diagnosis of Hypoparathyroidism. J Clin Endocrinol Metab. 2016;101(6):2284-99.

14. Underbjerg L, Sikjaer T, Mosekilde L, Rejnmark L. The Epidemiology of Nonsurgical Hypoparathyroidism in Denmark: A Nationwide Case Finding Study. J Bone Miner Res. 2015;30(9):1738-44.

15. Vadiveloo T, Donnan PT, Leese GP. A Population-Based Study of the Epidemiology of Chronic Hypoparathyroidism. J Bone Miner Res. 2018;33(3):47885.

16. Underbjerg L, Sikjaer T, Mosekilde L, Rejnmark L. Postsurgical hypoparathyroidism--risk of fractures, psychiatric diseases, cancer, cataract, and infections. J Bone Miner Res. 2014;29(11):2504-10.

17. Underbjerg L, Sikjaer T, Mosekilde L, Rejnmark L. Cardiovascular and renal complications to postsurgical hypoparathyroidism: a Danish nationwide controlled historic follow-up study. J Bone Miner Res. 2013;28(11):227785.

18. Sikjaer T, Moser E, Rolighed L, Underbjerg L, Bislev LS, Mosekilde L, et al. Concurrent Hypoparathyroidism Is Associated With Impaired Physical Function and Quality of Life in Hypothyroidism. J Bone Miner Res. 2016;31(7):1440-8.

19. Clarke BL. Bone disease in hypoparathyroidism. Arq Bras Endocrinol Metabol. 2014;58(5):545-52.

20. Cipriani C, Abraham A, Silva BC, Cusano NE, Rubin MR, McMahon DJ, et al. Skeletal changes after restoration of the euparathyroid state in patients with hypoparathyroidism and primary hyperparathyroidism. Endocrine. 2017;55(2):591-8.

21. Albright F, Ellsworth R. STUDIES ON THE PHYSIOLOGY OF THE PARATHYROID GLANDS: I. Calcium and Phosphorus Studies on a Case of Idiopathic Hypoparathyroidism. J Clin Invest. 1929;7 (2):183-201.

22. Sikjaer T, Amstrup AK, Rolighed L, Kjaer SG, Mosekilde L, Rejnmark L. PTH(1-84) replacement therapy in hypoparathyroidism: a randomized controlled trial on pharmacokinetic and dynamic effects after 6 months of treatment. J Bone Miner Res. 2013;28(10):2232-43.

23. Winer KK, Zhang B, Shrader JA, Peterson D, Smith M, Albert PS, et al. Synthetic human parathyroid hormone 1-34 replacement therapy: a randomized crossover trial comparing pump versus injections in the treatment of chronic hypoparathyroidism. J Clin Endocrinol Metab. 2012;97(2):391-9.

24. Mannstadt M, Clarke BL, Vokes T, Brandi ML, Ranganath L, Fraser WD, et al. Efficacy and safety of recombinant human parathyroid hormone (1-84) in hypoparathyroidism (REPLACE): a double-blind, placebo-controlled, randomised, phase 3 study. Lancet Diabetes Endocrinol. 2013;1(4):275-83.

25. Rubin MR, Cusano NE, Fan WW, Delgado Y, Zhang C, Costa AG, et al. Therapy of Hypoparathyroidism With PTH(1-84): A Prospective Six Year Investigation of Efficacy and Safety. J Clin Endocrinol Metab. 2016;101(7):2742-50.

26. Clarke BL, Vokes TJ, Bilezikian JP, Shoback DM, Lagast H, Mannstadt M. Effects of parathyroid hormone rhPTH(1-84) on phosphate homeostasis and vitamin D metabolism in hypoparathyroidism: REPLACE phase 3 study. Endocrine. 2017;55(1):273-82.

27. Cusano NE, Rubin MR, McMahon DJ, Zhang C, Ives R, Tulley A, et al. Therapy of hypoparathyroidism with PTH(1-84): a prospective four-year investigation of efficacy and safety. J Clin Endocrinol Metab. 2013;98(1):137-44.

28. Lakatos P, Bajnok L, Lagast H, Valkusz Z. An open-label extension study of parathyroid hormone rhpth(1-84) in adults with hypoparathyroidism. Endocr Pract. 2016;22(5):523-32.

29. Palermo A, Santonati A, Tabacco G, Bosco D, Spada A, Pedone C, et al. PTH(1-34) for Surgical Hypoparathyroidism: A 2-Year Prospective, OpenLabel Investigation of Efficacy and Quality of Life. J Clin Endocrinol Metab. 2018;103(1):271-80.

30. Cusano NE, Rubin MR, Irani D, Sliney J, Bilezikian JP. Use of parathyroid hormone in hypoparathyroidism. J Endocrinol Invest. 2013;36(11):1121-7.

31. Rosa RG, Barros AJ, de Lima AR, Lorenzi W, Da Rosa RR, Zambonato KD, et al. Mood disorder as a manifestation of primary hypoparathyroidism: a case report. J Med Case Rep. 2014;8:326.
32. Cusano NE, Rubin MR, McMahon DJ, Irani D, Anderson L, Levy E, et al. PTH(1-84) is associated with improved quality of life in hypoparathyroidism through 5 years of therapy. J Clin Endocrinol Metab. 2014;99(10):3694-

33. Cipriani C, Capriani C, Irani D, Bilezikian JP. Safety of osteoanabolic therapy: a decade of experience. J Bone Miner Res. 2012;27(12):2419-28.

34. Andrews EB, Gilsenan AW, Midkiff K, Sherrill B, Wu Y, Mann BH, et al. The US postmarketing surveillance study of adult osteosarcoma and teriparatide: study design and findings from the first 7 years. J Bone Miner Res. 2012;27(12):2429-37.

35. Cusano NE, Rubin MR, Bilezikian JP. PTH(1-84) replacement therapy for the treatment of hypoparathyroidism. Expert Rev Endocrinol Metab. 2015;10(1):5-13.

36. Cusano NE, Anderson L, Rubin MR, Silva BC, Costa AG, Irani D, et al. Recovery of parathyroid hormone secretion and function in postoperative hypoparathyroidism: a case series. J Clin Endocrinol Metab. 2013;98(11):428590.

37. Cusano NE, Rubin MR, Zhang C, Anderson L, Levy E, Costa AG, et al. Parathyroid hormone 1-84 alters circulating vascular endothelial growth factor levels in hypoparathyroidism. J Clin Endocrinol Metab. 2014;99(10):E2025-8.

38. Rubin MR, Zwahlen A, Dempster DW, Zhou H, Cusano NE, Zhang C, et al Effects of Parathyroid Hormone Administration on Bone Strength in Hypoparathyroidism. J Bone Miner Res. 2016;31(5):1082-8.

39. Zea J, Londoño S, Cardona E, Álvarez D, Gómez J, Rúa C, et al. Resultados preliminares de la caracterización del hipoparatiroidismo en el Hospital Universitario San Vicente Fundación. Revista Colombiana de Endocrinología, Diabetes y Metabolismo. 2018;5(2):86-7.

40. Sikjaer T, Rejnmark L, Rolighed L, Heickendorff L, Mosekilde L, Group HS The effect of adding PTH(1-84) to conventional treatment of hypoparathyroidism: a randomized, placebo-controlled study. J Bone Miner Res. 2011;26(10):2358-70.

41. Rubin MR, Sliney J, McMahon DJ, Silverberg SJ, Bilezikian JP. Therapy of hypoparathyroidism with intact parathyroid hormone. Osteoporos Int. 2010;21(11):1927-34.

42. Rubin MR, Dempster DW, Sliney J, Zhou H, Nickolas TL, Stein EM, et al PTH(1-84) administration reverses abnormal bone-remodeling dynamics and structure in hypoparathyroidism. J Bone Miner Res. 2011;26(11):272736.

43. Bilezikian JP, Clarke BL, Mannstadt M, Rothman J, Vokes T, Lee HM, et al. Safety and Efficacy of Recombinant Human Parathyroid Hormone in Adults With Hypoparathyroidism Randomly Assigned to Receive Fixed 25- $\mu \mathrm{g}$ or 50- $\mu$ g Daily Doses. Clin Ther. 2017;39(10):2096-102.

44. Vokes TJ, Mannstadt M, Levine MA, Clarke BL, Lakatos P, Chen K, et al. Recombinant Human Parathyroid Hormone Effect on Health-Related Quality of Life in Adults With Chronic Hypoparathyroidism. J Clin Endocrinol Metab. 2018;103(2):722-31.

45. Winer KK, Sinaii N, Reynolds J, Peterson D, Dowdy K, Cutler GB. Long-term treatment of 12 children with chronic hypoparathyroidism: a randomized trial comparing synthetic human parathyroid hormone $1-34$ versus calcitriol and calcium. J Clin Endocrinol Metab. 2010;95(6):2680-8.

46. Winer KK, Sinaii N, Peterson D, Sainz B, Cutler GB. Effects of once versus twice-daily parathyroid hormone 1-34 therapy in children with hypoparathyroidism. J Clin Endocrinol Metab. 2008;93(9):3389-95.

47. Winer KK, Ko CW, Reynolds JC, Dowdy K, Keil M, Peterson D, et al. Long-term treatment of hypoparathyroidism: a randomized controlled study comparing parathyroid hormone-(1-34) versus calcitriol and calcium. J Clin Endocrinol Metab. 2003;88(9):4214-20.

48. Winer KK, Yanovski JA, Sarani B, Cutler GB. A randomized, cross-over tria of once-daily versus twice-daily parathyroid hormone 1-34 in treatment of hypoparathyroidism. J Clin Endocrinol Metab. 1998;83(10):3480-6.

49. Winer KK, Yanovski JA, Cutler GB. Synthetic human parathyroid hormone 1-34 vs calcitriol and calcium in the treatment of hypoparathyroidism. JAMA. 1996;276(8):631-6.

50. Sikjaer T, Rejnmark L, Thomsen JS, Tietze A, Brüel A, Andersen G, et al. Changes in 3-dimensional bone structure indices in hypoparathyroid patients treated with PTH(1-84): a randomized controlled study. J Bone Miner Res. 2012;27(4):781-8.

51. Sikjaer T, Rolighed L, Hess A, Fuglsang-Frederiksen A, Mosekilde L, Rejnmark L. Effects of PTH(1-84) therapy on muscle function and quality of life in hypoparathyroidism: results from a randomized controlled trial. Osteoporos Int. 2014;25(6):1717-26.

52. Santonati A, Palermo A, Maddaloni E, Bosco D, Spada A, Grimaldi F, et al. PTH(1-34) for Surgical Hypoparathyroidism: A Prospective, Open-Label Investigation of Efficacy and Quality of Life. J Clin Endocrinol Metab. 2015;100(9):3590-7. 